\title{
Involvement of cytokinins in STOP1- mediated resistance to proton toxicity
}

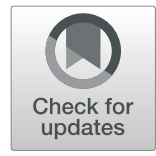

Fei Jiang ${ }^{1,2}$, Sangbom M. Lyi ${ }^{1}$, Tianhu Sun ${ }^{3}$, Li Li $^{1,3}$, Tao Wang $^{2}$ and Jiping Liu ${ }^{1,3^{*}}$ (D)

\begin{abstract}
STOP1 (sensitive to proton rhizotoxicity 1 ) is a master transcription factor that governs the expression of a set of regulatory and structural genes involved in resistance to aluminum and low pH (i.e., proton) stresses in Arabidopsis. However, the mechanisms and regulatory networks underlying STOP1-mediated resistance to proton stresses are largely unclear. Here, we report that low-pH stresses severely inhibited root growth of the stop 1 plants by suppressing root meristem activities. Interestingly, the stop1 plants were less sensitive to exogenous cytokinins at normal and low pHs than the wild type. Significantly, low concentrations of cytokinins promoted root growth of the stop 1 mutant under low-pH stresses. Moreover, lateral and adventitious root formation was stimulated in stop 1 and by low-pH stresses but suppressed by cytokinins. Further studies of the expression patterns of a cytokinin signaling reporter suggest that both the loss-of-function mutation of STOP1 and low-pH stresses suppressed cytokinin signaling outputs in the root. Furthermore, the expression of critical genes involved in cytokinin biosynthesis, biodegradation, and signaling is altered in the stop 1 mutant in response to low-pH stresses. In conclusion, our results reveal a complex network of resistance to low-pH stresses, which involves coordinated actions of STOP1, cytokinins, and an additional low-pH-resistant mechanism for controlling root meristem activities and root growth upon proton stresses.
\end{abstract}

Keywords: Arabidopsis, Cytokinin, Cytokinin signaling output, Low pH stress, Proton stress, Resistance, STOP1, Tolerance

\section{Highlight}

We demonstrate that low-pH (proton) stresses and STOP1 functions affect cytokinin signaling outputs, which in turn regulate root growth. Cytokinins play crucial roles in STOP1-mediated resistance to proton stresses.

\section{Introduction}

In Arabidopsis, STOP1 (sensitive to proton rhizotoxicity) encodes a master transcription factor that regulates a set of genes critical for resistance to a range of abiotic stresses, including aluminum (Al) toxicity and low-pH (i.e., proton) stresses (Iuchi et al., 2007; Koyama et al.,

\footnotetext{
* Correspondence: j1233@cornell.edu

${ }^{1}$ Robert W. Holley Center for Agriculture and Health, USDA-ARS, Ithaca, NY 14853, USA

${ }^{3}$ Plant Breeding and Genetics Section, School of Integrative Plant Science, Cornell University, Ithaca, NY 14853, USA

Full list of author information is available at the end of the article
}

2021; Sadhukhan et al., 2021). Therefore, the loss-offunction stop 1 mutants are susceptible to low-pH and $\mathrm{Al}$ stresses (Iuchi et al., 2007; Liu et al., 2009). Although it has been well documented that STOP1 confers $\mathrm{Al}$ resistance via controlling the expression of a set of $\mathrm{Al}$ resistant genes, including ALMT1, MATE, and ALS3 (Liu et al., 2009; Sawaki et al., 2009), the mechanisms underlying STOP1-mediated resistance to proton stresses remain unclear (Liu et al., 2009; Liu et al., 2012).

Cytokinins are a class of phytohormones that regulate various developmental and physiological processes, including cell division, shoot and root development, and responses to pathogens (Mok and Mok, 2001; Werner et al., 2003; Müller, 2011; Kieber and Schaller, 2018). Although cytokinins control cell proliferation in meristems, their actions in the root and shoot meristems are opposite (Werner et al., 2001; Werner et al., 2003). Cytokinins are positive regulators of cell division and 
meristem activities in the shoot apical meristem. Thus, they promote shoot growth and the formation of inflorescence meristems and leaf primordia (Werner et al., 2003). In contrast, cytokinins negatively regulate root growth and development as they suppress cell division in the root apical meristem and inhibit the formation of lateral root and adventitious root primordia (Werner et al., 2003; Dello Ioio et al., 2007). Lateral roots emerge from the root pericycle, while adventitious roots arise from an organ other than the root, such as a stem.

In plants, cytokinin homeostasis is maintained by de novo biosynthesis, import/export, formation of conjugates, and catabolism (Mok and Mok, 2001). In Arabidopsis, the cytokinin-mediated responsive and regulatory functions are facilitated via a two-component signaling (TCS) system where the initial cytokinin signals are perceived by the membrane-localized two-component histidine-kinase receptors, AHK2, AHK3, and AHK4 (AHK, Arabidopsis Histidine Kinase). Then, the signals are further transduced through a series of His-Asp phosphorelay events that lead to the activation of a set of transcription factors in the nucleus, which modulates the expression and function of different response regulators (RRs), leading to the final realization of the cytokinin signaling (Hwang and Sheen, 2001; Ferreira and Kieber, 2005; To and Kieber, 2008; Cheng and Kieber, 2014).

Although cytokinins have been implicated in plant resistance to abiotic stresses, including cold, osmotic, drought, and salt stresses (Tran et al., 2007; Argueso et al., 2009; Ha et al., 2012; O'Brien and Benková, 2013; Zwack and Rashotte, 2015), their roles in resistance to low-pH stresses remain unknown. Here, we report that both STOP1 functions and proton stresses suppress cytokinin signaling outputs in the root, influencing root growth under low-pH stresses. Therefore, our results reveal a complex network involving cytokinins in STOP1mediated resistance to low-pH stresses in Arabidopsis.

\section{Results}

\section{Loss-of-STOP1 function and low-pH stresses reduce root} sensitivity to cytokinin inhibition

At normal $\mathrm{pH}(5.8,+0 \mu \mathrm{M} \mathrm{BA}), 5$-d-old seedlings of the wild type (WT, Col-O) and the stop1 null mutant (SALK_114180) exhibited a similar root-growth pattern (Fig. 1A, the left panel), confirming that STOP1 is not required for normal plant growth (Iuchi et al., 2007). However, a low-pH treatment $(\mathrm{pH} 4.3,+0 \mu \mathrm{M}$ BA) reduced the WT root growth by $31 \%$ (Fig. 1A, the left panel) but inhibited root growth of the stop1 mutant by $\sim 95 \%$ (Fig. 1A, the left panel), confirming that the stop 1 mutant is highly susceptible to proton stresses (Iuchi et al., 2007; Sawaki et al., 2009). These results indicate that STOP1 plays an essential role in resistance to low$\mathrm{pH}$ stresses, i.e., proton stresses, in Arabidopsis.

Next, we examined the combinational effects of proton stresses and 6-benzyladenine (BA), an active cytokinin (Flores and Tobin, 1986), on root growth of WT and stop1. Under a pH 5.8 condition, root growth of the WT and the stop 1 mutant was inhibited comparably by low BA concentrations $(\leq 0.01 \mu \mathrm{M})$ (Fig. 1B). The relative root growth (RRG\%; +BA vs. $-\mathrm{BA}$, at $\mathrm{pH}$ 5.8) of the WT and stop 1 plants was comparably decreased by $\sim 5$ and $16 \%$ in the presence of 0.005 and $0.010 \mu \mathrm{M} \mathrm{BA}$, respectively (Fig. 1B). However, higher BA concentrations $(\geq 0.5 \mu \mathrm{M}, \mathrm{pH} 5.8)$ caused more potent root-growth inhibition in the WT than in the stop1 mutant (Fig. 1A, the right panel, and 1B). The root growth was decreased by 73 and $61 \%$ in the WT and stop 1 plants, respectively, in the presence of $0.5 \mu \mathrm{M} \mathrm{BA}$ at $\mathrm{pH} 5.8$ (Fig. 1A, B). These results indicate that the stop1 mutant was less sensitive to cytokinin-induced root-growth inhibition at $\mathrm{pH}$ 5.8.

Interestingly, the WT became insensitive to low concentrations of BA $(\leq 0.01 \mu \mathrm{M})$, i.e., $\mathrm{RRG} \% \mathrm{~s}=100 \%$, at $\mathrm{pH}$ 4.3 (Fig. 1C), whereas the same low BA concentrations $(\leq 0.01 \mu \mathrm{M})$ led to slightly reduced root growth of the WT plants at pH 5.8 (Fig. 1B). Furthermore, although higher BA concentrations $(\geq 0.5 \mu \mathrm{M})$ also strongly inhibited WT root growth at $\mathrm{pH} 4.3$ (Fig. 1C), the degrees of root-growth inhibition were less profound at $\mathrm{pH} 4.3$ than at pH 5.8 (Fig. 1B, C). In WT, RRG\%'s (+BA vs. -BA) decreased by $73 \%$ at pH 5.8 vs. $69 \%$ at pH 4.3 (Fig. 1B, $\mathrm{C})$. These results indicate that proton stresses reduced the sensitivity of the WT to cytokinins.

More interestingly, at low $\mathrm{pH}$ (4.3), low BA concentrations $(0.005,0.01$, and $0.5 \mu \mathrm{M})$ promoted root growth of the stop 1 mutant, leading to 1.4-, 1.6-, and 1.7-fold increases in RRG\%'s (+BA vs. $-\mathrm{BA}, \mathrm{pH} 4.3$ ), respectively (Fig. 1A, C). However, when BA concentrations were $\geq$ $2 \mu \mathrm{M}$, the promotional effect of BA on root growth of stop1 disappeared (Fig. 1C). These results further support the notion that proton stresses suppress the cytokinins' inhibitory impacts on root growth in Arabidopsis.

\section{Involvement of proton stresses, STOP1 function, and cytokinins in controlling root-meristem size and root stem-cell identity}

Root growth results from cell division in the root meristem and subsequent cell elongation in the root elongation zone. It has been demonstrated that cytokinins play crucial roles in regulating root-meristem activities (Dello Ioio et al., 2007). To investigate a possible interaction of low-pH stresses, STOP1 function, and exogenous cytokinins in controlling Arabidopsis root-meristem activities, we followed the root-meristem development of 


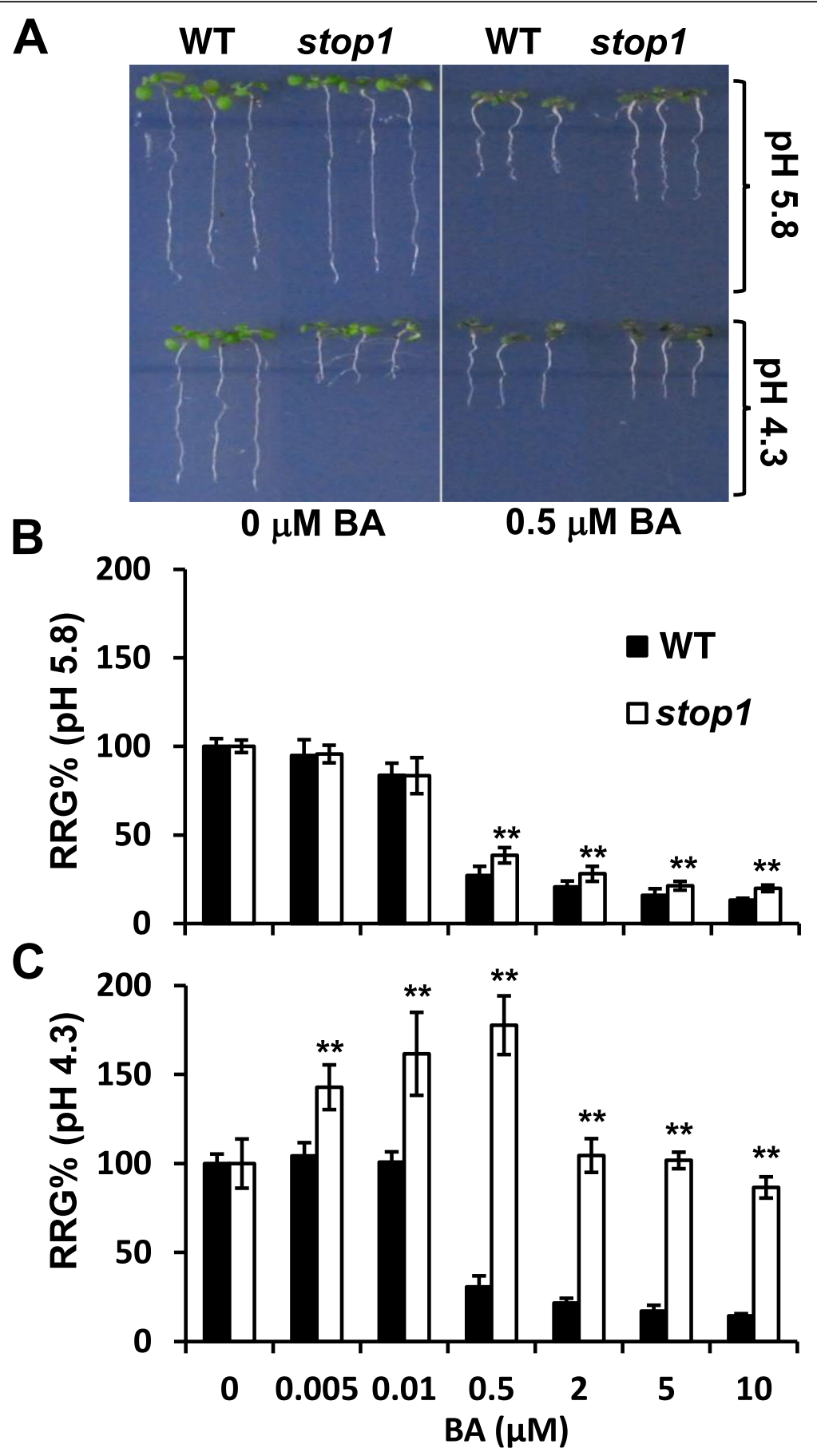

Fig. 1 The stop 1 mutant is hyposensitive to cytokinins and is partially rescued by low concentrations of BA at pH 4.3. A Representative 5-d-old seedlings of WT and stop 1 germinated and grown on $1 / 2 \times$ MS gellan gum plates ( $\mathrm{pH} 4.3$ or 5.8) supplemented with or without $0.5 \mu \mathrm{M}$ BA. Relative root growth (RRG\%) (+BA vs. -BA) of WT and stop1 at pH 5.8 (B) and pH 4.3 (C). Data are means \pm SD of three biological replicates. In each replicate, $n=20$. Asterisks indicate significant differences $(p<0.01)$ between stop1 and WT at indicated BA concentrations

the WT and stop1 mutant under different $\mathrm{pH}$ and cytokinin treatments.

Root-meristem size, or root-meristem-cell number, is defined as the number of a file of cortex cells from the quiescent center (QC) to the first elongated cell (Fig. 2A)
(Casamitjana-Martınez et al., 2003; Dello Ioio et al., 2007). The first elongated cell also marks the root transition zone (TZ) (Baluška et al., 2010). At pH 5.8 (-BA), the rootmeristem size was comparable between the 4-d-old WT and stop 1 seedlings (Fig. 2A, B). However, a $16 \mathrm{~h}$ 

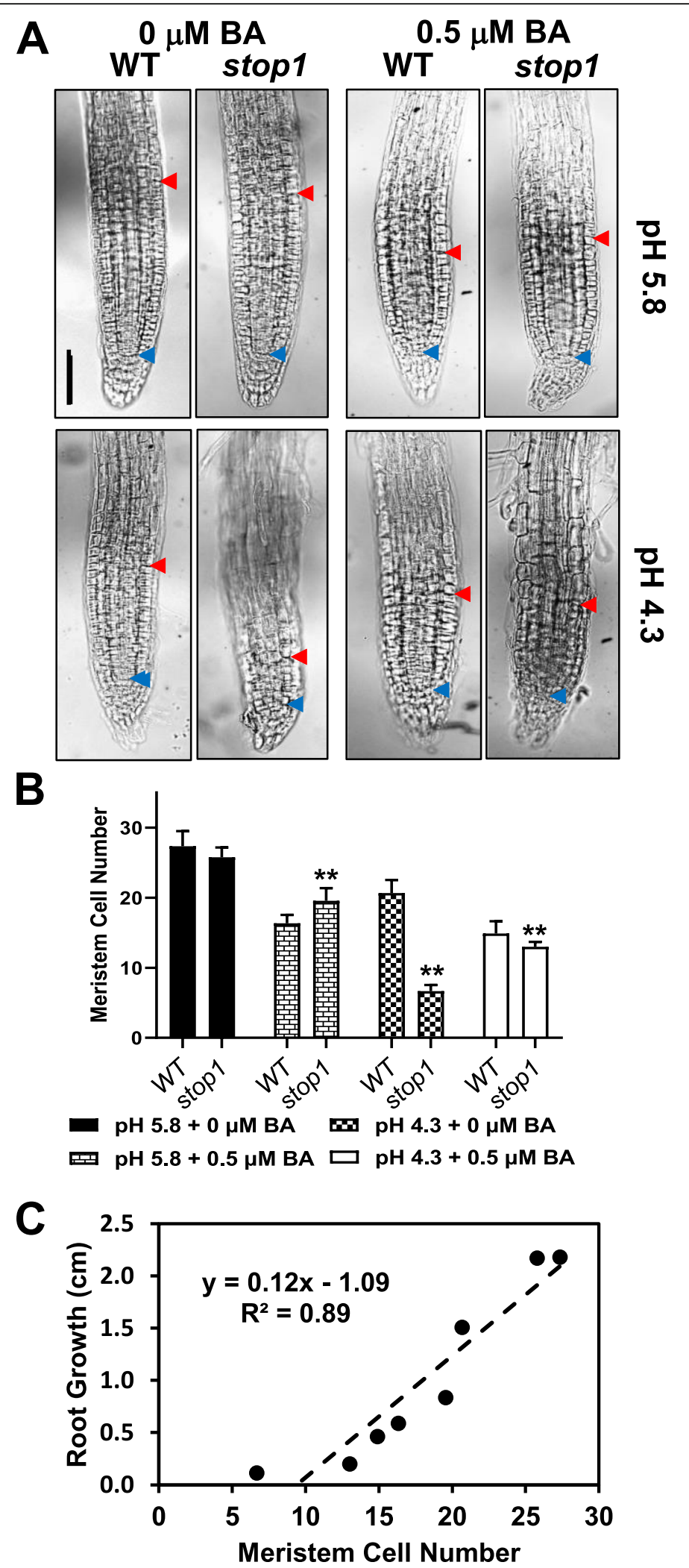

Fig. 2 (See legend on next page.) 
(See figure on previous page.)

Fig. 2 STOP1 function and low-pH stresses affect root meristem cell size (A, B). WT and stop 1 seeds were germinated and grown on $1 / 2 \times$ MS agar plates for $4 \mathrm{~d}$. Then, the 4 - $d$-old seedlings were transferred to new $1 / 2 \times$ MS gellan gum plates ( $\mathrm{pH} 5.8$ or 4.3 ) supplemented with or without $0.5 \mu \mathrm{M}$ BA for a 16-h treatment. Root meristem size is defined as the number of cells from the quiescent center (QC) to the first elongated cortex cell. Data represent the mean of 25 plants $(n=25)$. C Linear regression analysis for root growth and meristem-stem cell number. Each dot represents the mean from ten plants of the WT or stop 1 under different treatments. Scale bar $=100 \mu \mathrm{m}$. The blue arrowhead point to the QC; the red arrowhead indicates the root transition zone (TZ) marked by the first elongated cortex cell. Asterisks represent significant differences between WT and stop 1 under indicated treatment concentrations $(P<0.01)$

treatment of $0.5 \mu \mathrm{M}$ BA significantly reduced rootmeristem size in both lines at pH 5.8 (Fig. 2A, B). This result was consistent with the reported cytokinin's action on root meristem size (Dello Ioio et al., 2007). In contrast, the exogenous cytokinin had significantly less influence on the root meristem size of the stop1 mutant than the WT at pH 5.8 (Fig. 2A, B). The root meristem size decreased by $40 \%$ in WT versus $24 \%$ in stop 1 upon a $0.5 \mu \mathrm{M}$ BA treatment at pH 5.8 (Fig. 2B). This result indicates that the loss-of-STOP1 function suppresses the inhibitory effects of cytokinins on root meristem activities.

At low pH (4.3) without an exogenous BA, the WT root meristem size decreased by $24 \%$ compared with that at pH 5.8 (-BA) (Fig. 2A, B). This result indicates that the low-pH stresses inhibit root meristem activities in Arabidopsis. However, the WT still maintained an intact root stem-cell niche at pH 4.3 (Fig. 2A; Fig. S2A). In contrast, the root meristem activity was severely suppressed in the stop1 mutant at $\mathrm{pH} 4.3$, decreased by $74 \%$ compared with the pH 5.8 (-BA) condition (Fig. 2A, B). Furthermore, the stop 1 mutant lost the root quiescent center (QC) and the root stem-cell niche, at $\mathrm{pH} 4.3$ (-BA) (Fig. 2A; Fig. S2B). These results indicate that STOP1 is essential for maintaining root meristem activities and root stem-cell identity under low-pH stresses.

Interestingly, a $0.5 \mu \mathrm{M}$ BA treatment improved root meristem activities of the stop1 plant at $\mathrm{pH} 4.3$ (Fig. 2A, B). The root meristem size of stop1 increased $95 \%$ and the root stem-cell niche appeared with the BA treatment compared to the non-BA treatment condition at $\mathrm{pH} 4.3$ (Fig. 2A, B; Fig. S2C). Therefore, the effect of $0.5 \mu \mathrm{M}$ BA application on partially restored root growth of stop 1 at pH 4.3 (Fig. 1A, C) could be partly explained by the BAmediated restoration of root stem cells and the improved root meristem activities.

Moreover, compared with the non-BA condition, a $0.5 \mu \mathrm{M}$ BA treatment resulted in a $24 \%$ decrease in root meristem size of the WT plant at $\mathrm{pH} 4.3$, compared with a $40 \%$ decrease at pH 5.8 (Fig. 2A, B). These results indicated that the low-pH stress reduced the inhibitory effect of exogenous BA on root meristem activities.

Next, we conducted a statistical analysis to investigate the relationships between root growth, root-meristem size, low-pH stresses, and STOP1 function. Regression analyses indicated that root growth was highly associated with the root-meristem size of the WT and stop1 plants under different $\mathrm{pH}$ and BA treatments $\left(R^{2}=0.89\right)$, which means $89 \%$ of the root-growth variation could be explained by the variation of the root-meristem size (Fig. $2 \mathrm{C})$. Therefore, our results suggest that root-meristem activities mainly determined the observed root growth under low-pH stresses.

\section{The stop 1 mutant produces more lateral roots and adventitious roots}

We further evaluated the combinational effects of cytokinins, $\mathrm{pH}$, and STOP1 functions on lateral-root and adventitious-root formation in Arabidopsis. At $\mathrm{pH} 5.8$ (-BA), the lateral-root density (i.e., lateral-root number per $\mathrm{cm}$ of the primary root of a plant) and adventitiousroot density (i.e., adventitious-root number per plant) of the 5-d-old WT seedlings were 0.1 and 0.4 , respectively (Table 1). In contrast, the stop 1 plants possessed 0.8 and 1.2 lateral-root and adventitious-root density under the same condition, respectively, significantly higher than the WT (Table 1). These results indicated that the lossof-function mutation of STOP1 promoted the lateraland adventitious-root formation at $\mathrm{pH}$ 5.8.

Furthermore, compared with the pH 5.8 (-BA) treatment, the low-pH $(4.3,-\mathrm{BA})$ treatment significantly increased the numbers of lateral-root density in both WT and stop1 (Table 1). The lateral-root density increased to 0.7 and 40.0 in WT and stop1, respectively. These results indicated that compared with pH 5.8 (-BA), the low-pH (4.3, -BA) treatment caused a 7 - and 50-fold increase in lateral-root density in the WT and the stop 1 mutant, respectively (Table 1 ). These results indicate that both the loss-of-function of STOP1 and low-pH stresses promote lateral root formation in Arabidopsis. However, the effect of low $\mathrm{pH}$ on lateral-root formation was much more profound in stop1 than in WT.

For adventitious roots, a $\mathrm{pH}$ change from 5.8 to 4.3 slightly increased the adventitious-root density in both WT and stop1 (Table 1). However, the stop1 mutant possessed significantly higher adventitious-root density than the WT under both normal- and low-pH conditions. Taken together, these results indicated that the STOP1 function imposed a more profound impact on adventitious-root formation than the effect of $\mathrm{pH}$ changes. 
Table 1 Lateral-root density (LRD) and adventitious-root density (ARD) of seedlings treated without (-) or with (+) $0.5 \mu \mathrm{M} B A$ at pH 5.8 or 4.3 on agar plates at six days after germination (DAG)

\begin{tabular}{|c|c|c|c|c|c|c|}
\hline & \multicolumn{2}{|c|}{ Lateral root density $(-\mathrm{BA})$} & \multirow[b]{2}{*}{$P$ value } & \multicolumn{2}{|c|}{ Adventitious root density (-BA) } & \multirow[b]{2}{*}{$P$ value } \\
\hline & WT & stop1 & & WT & stop1 & \\
\hline pH 5.8 & $0.1 \pm 0.2$ & $0.8 \pm 0.4$ & $<0.001$ & $0.4 \pm 0.5$ & $1.2 \pm 0.4$ & $<0.01$ \\
\hline \multirow[t]{3}{*}{ pH 4.3} & $0.7 \pm 0.4$ & $40.0 \pm 4.7$ & $<0.001$ & $0.5 \pm 0.3$ & $1.4 \pm 0.5$ & $<0.01$ \\
\hline & \multicolumn{2}{|c|}{ Lateral root density $(+B A)$} & & \multicolumn{2}{|c|}{ Adventitious root density (+BA) } & \\
\hline & WT & stop 1 & $P$ value & WT & stop1 & $P$ value \\
\hline pH 5.8 & $0.0 \pm 0.0$ & $0.0 \pm 0.0$ & 1 & $0.0 \pm 0.0$ & $0.8 \pm 0.6$ & $<0.01$ \\
\hline pH 4.3 & $0.0 \pm 0.0$ & $0.0 \pm 0.0$ & 1 & $0.0 \pm 0.0$ & $0.9 \pm 0.3$ & $<0.001$ \\
\hline
\end{tabular}

Lateral root density is defined as the number of lateral roots per $\mathrm{cm}$ of a primary root of a plant. Adventitious root density is defined as the number of adventitious roots per plant. Data are means \pm SD of three replicates. In each replicate, $n=10$. The $t$-tests were conducted to compare the differences between WT and stop1. BA, 6-benzyladenine

\section{Suppression of lateral- and adventitious-root formation by cytokinins}

We then proceeded to test the effects of cytokinins on the lateral-root and adventitious-root formation in WT and stop1. Supplementation of $0.5 \mu \mathrm{M}$ BA to the growth medium abolished the lateral-root formation in WT under both pH 5.8 and 4.3 conditions (Table 1). In contrast, treatment of $0.5 \mu \mathrm{M} \mathrm{BA}$ slightly reduced the adventitious-root density to 0.8 and 0.9 in the stop $1 \mathrm{mu}-$ tant at pH 5.8 and 4.3, respectively (Table 1 ). These results indicated that the loss-of-function of STOP1 partially offset the inhibitory effects of $0.5 \mu \mathrm{M}$ BA on adventitious root formation.

Low-pH stresses and STOP1 functions influence cytokinin signaling outputs of the Arabidopsis plants

The different responses of the WT and stop1 mutant to exogenous BA at pH 5.8 and 4.3 (Figs. 1, 2; and Table 1) prompted us to investigate the possible involvement of cytokinin signaling in STOP1-mediated resistance to low-pH stresses. We used the cytokinin-sensitive twocomponent-output-sensor (new) (TCSn) reporter to monitor cytokinin-signaling outputs in Arabidopsis plants in vivo. The synthetic TCSn promoter contains Btype Arabidopsis response regulator (ARR)-binding motifs and a minimal $35 \mathrm{~S}$ promoter, explicitly responding to cytokinins but not other plant hormones (Müller and Sheen, 2008; Zürcher et al., 2013).

We examined the TCSn:GFP expression patterns in transgenic WT and stop1 plants stably transformed with the TCSn:GFP construct. In general, 4-d-old WT and stop1 seedlings shared the same tissue-specific TCSn:GFP expression patterns except that the TCSn:GFP expression intensity was weaker in the stop1 mutant than in WT (Fig. 3).

At pH 5.8 (-BA), TCSn:GFP was expressed strongly in the root maturation zone with the most robust expression in the region near the root-shoot junction (Fig. 3A, B). Moreover, TCSn:GFP was strongly expressed in the root cap but weakly manifested in the stele precursor in the root meristem area (Fig. 3A, B, I, J).

At $\mathrm{pH} 5.8$, a $16-\mathrm{h} 0.5 \mu \mathrm{M}$ BA treatment significantly enhanced the TCSn:GFP fluorescent intensity of the WT and stop1 (Fig. 3C, D, K, L). Moreover, the TCSn:GFP expression was expanded to the root distal-elongation zone close to the root meristem region as well as the vasculature of the shoot (Fig. 3C, D). In addition, the TCSn:GFP expression was intensified in the root tip, especially in the root meristem region (Fig. $3 \mathrm{C}, \mathrm{D}, \mathrm{K}, \mathrm{L}$ ). However, the TCSn:GFP intensity in the root tip was weaker in stop1 than in WT (Fig. 3C, D, K, L).

Interestingly, the low-pH $(4.3,-\mathrm{BA})$ treatment significantly brought down the TCSn:GFP expression in WT and stop1 (Fig. 3E, F, M, N) compared with that of the corresponding lines at pH 5.8 (-BA) (Fig. 3A, B, I, J). Furthermore, the TCSn:GFP fluorescence was weaker in stop1 (Fig. 3B, F, J, N) than in WT (Fig. 3A, E, I, M) under the low-pH $(4.3,-\mathrm{BA})$ condition.

At low $\mathrm{pH}(4.3)$, a $0.5 \mu \mathrm{M}$ BA treatment led to enhanced TCSn:GFP fluorescence of the WT (Fig. 3G, $\mathrm{O})$ and stop1 (Fig. 3H, P) compared with the corresponding non-BA treatment (Fig. 3E, F, M, N). However, the TCSn:GFP fluorescence was weaker at $\mathrm{pH}$ 4.3 (Fig. 3G, H, O, P) than at pH 5.8 (Fig. 3C, D, K, $\mathrm{L}$ ) and weaker in stop 1 (Fig. 3D, H, L, P) than in WT (Fig. 3C, G, K, O).

Taken together, the TCSn:GFP fluorescence intensity, or the cytokinin signaling output, is more robust in the WT than in the stop1 mutant and stronger at $\mathrm{pH} 5.8$ than at low $\mathrm{pH}$. These results suggest that low-pH stresses and the loss-of-function STOP1 suppress cytokinin-signaling outputs in Arabidopsis.

\section{Expression of key genes involved in cytokinin} biosynthesis, degradation, and signaling under low-pH stresses

To further address the roles of cytokinins in STOP1mediated low-pH resistance, we examined the expression 


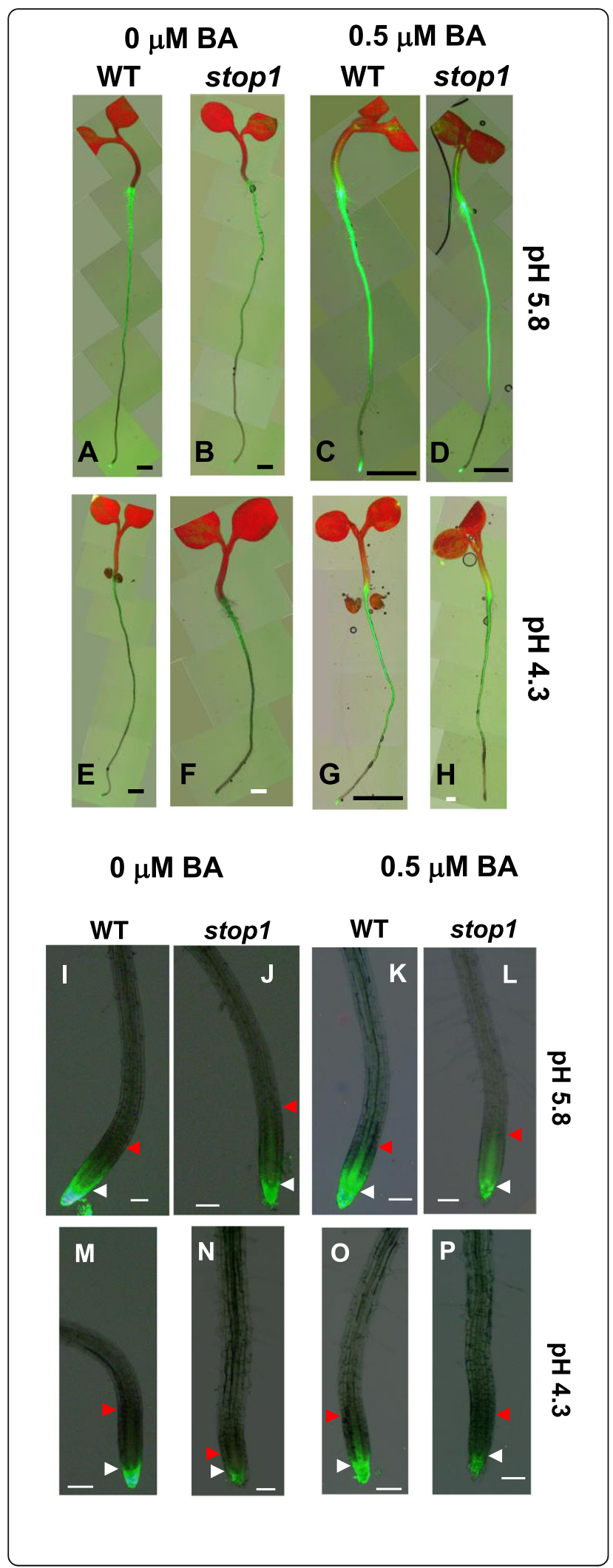

Fig. 3 Cytokinin signaling outputs manifested by the TCSn:GFP fluorescence of 4-d-old seedlings (A-H) and their root tips (I-P) of the WT and stop1. Seeds were germinated on $1 / 2 \times$ MS agar plates (pH 5.8) for $4 \mathrm{~d}$. Then, 4-d-old seedlings were transferred onto $1 / 2 \times \mathrm{MS}$ gellan gum plates ( $\mathrm{pH} 5.8$ or 4.3 ) with or without supplementation of $0.5 \mu \mathrm{M}$ BA for an additional 16-h treatment. Scale bars represent $0.1 \mathrm{~cm}$ for AE, G; $0.01 \mathrm{~cm}$ for $\mathrm{F}, \mathrm{H} ; 100 \mu \mathrm{m}$ for I-P

patterns of critical genes in the TCS system, including those encoding the enzymes for cytokinin biosynthesis (IPT3 and CYP735A2) and degradation (CKX1) as well as cytokinin receptors (AHK2, AHK3, and AHK4) and typeA Arabidopsis response regulators (ARRs).

Adenosine phosphate-isopentenyltransferases (IPT) and cytochrome P450 mono-oxygenases CYP735A1 and CYP735A2 are critical enzymes of cytokinin biosynthesis (Hirose et al., 2008). Cytokinin oxidase/dehydrogenases (CKX; EC 4.5.99.12) are primary enzymes responsible for irreversible degradation of cytokinins and the majority of metabolic cytokinin inactivity (Mok and Mok, 2001). The cytokinin receptors AHK2, AHK3, and AHK4 are partially redundant positive elements in cytokine sensing (Ferreira and Kieber, 2005; Brenner et al., 2012; Cheng and Kieber, 2014). AHK3 has been demonstrated to regulate the differentiation of root meristem cells in Arabidopsis (Perilli et al., 2013). In contrast, the type-A ARRs are the primary cytokinin-responsive elements that negatively influence the cytokinin signaling outputs (Hwang and Sheen, 2001; Kiba et al., 2003; To et al., 2004; Leibfried et al., 2005).

Real-time RT-qPCR analyses indicated that IPT3 and CKX1 expression levels in the root were comparable between WT and stop 1 under the control condition $(0 \mathrm{~h}$, pH 5.8) (Fig. 4A, C). However, CYP735A2 expression was significantly higher in stop1 than in WT under the control condition ( $0 \mathrm{~h}, \mathrm{pH}$ 5.8) (Fig. 4B). The expression of IPT3, CYP735A2, and CKX1 was significantly enhanced and peaked after $12 \mathrm{~h}$ low-pH (4.3) treatment in both WT and stop1 (Fig. 4). However, the induction of the cytokinin-promoting genes, IPT3 and CYP735A2, occurred much earlier, i.e., at $2 \mathrm{~h}$, in stop 1 than in WT whose strong IPT3 and CYP735A2 induction occurred at $12 \mathrm{~h}$ after the low-pH treatment (Fig. 4A, B). After peaked at $12 \mathrm{~h}$ after the low-pH treatment, IPT3 and CYP735A2 expression dropped back to their basal levels at $24 \mathrm{~h}$ in both WT and stop1 (Fig. 4A, B).

In contrast, expression of the cytokinin biodegradation gene, $C K X 1$, in the root was initially suppressed by $2 \mathrm{~h}$ low-pH treatment but strongly induced and peaked at $12 \mathrm{~h}$ in both WT and stop1 (Fig. 4C). At $24 \mathrm{~h}$, CKX1 expression dropped back to its basal level in the WT but remained at a high level in stop1 (Fig. 4C).

Under the control condition ( $\mathrm{pH} 5.8,0 \mathrm{~h}$ ), the expression of the cytokinin receptor gene, $A H K 3$, in the root was 

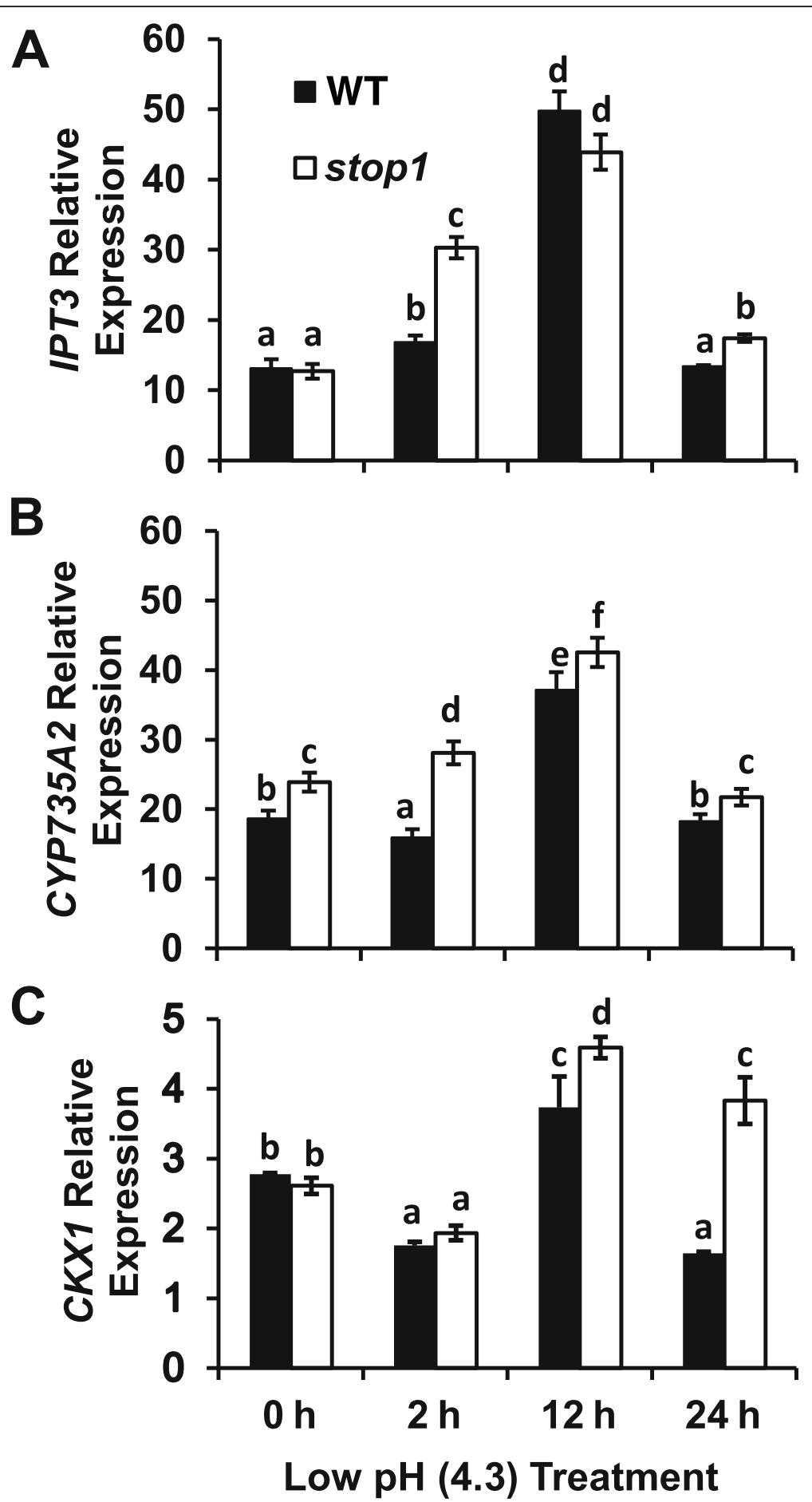

Fig. 4 Relative expression of IPT3 (A), CYP735A2 (B), and CKXI (C) after low pH (4.3) treatment. Seeds were germinated and grown hydroponically in $1 / 2 \times$ MS solution ( $\mathrm{pH}$ 5.8) for $5 \mathrm{~d}$ before the seedlings were transferred to a fresh low-pH (4.3) hydroponic growth solution. Total RNAs were extracted from the root tissues for real-time RT-qPCR analyses. Data are means \pm SD $(n=3)$. *, significant differences between the stop 1 mutant and WT $(p<0.05)$, **, highly significant differences between the stop 1 mutant and the WT $(p<0.01)$

comparable between the WT and the stop1 mutant (Fig. 5A). However, after $2 \mathrm{~h}$ low-pH treatment, $A H K 3$ expression in the root was quickly and strongly induced in stop1 but was slightly enhanced in the WT (Fig. 5A). In addition, $A H K 3$ expression peaked at $12 \mathrm{~h}$ in both the WT and the stop 1 mutant (Fig. 5A). However, at $24 \mathrm{~h}, A H K 3$ expression declined to its basal level in the WT but remained at a high level in stop1 (Fig. 5A). These results 


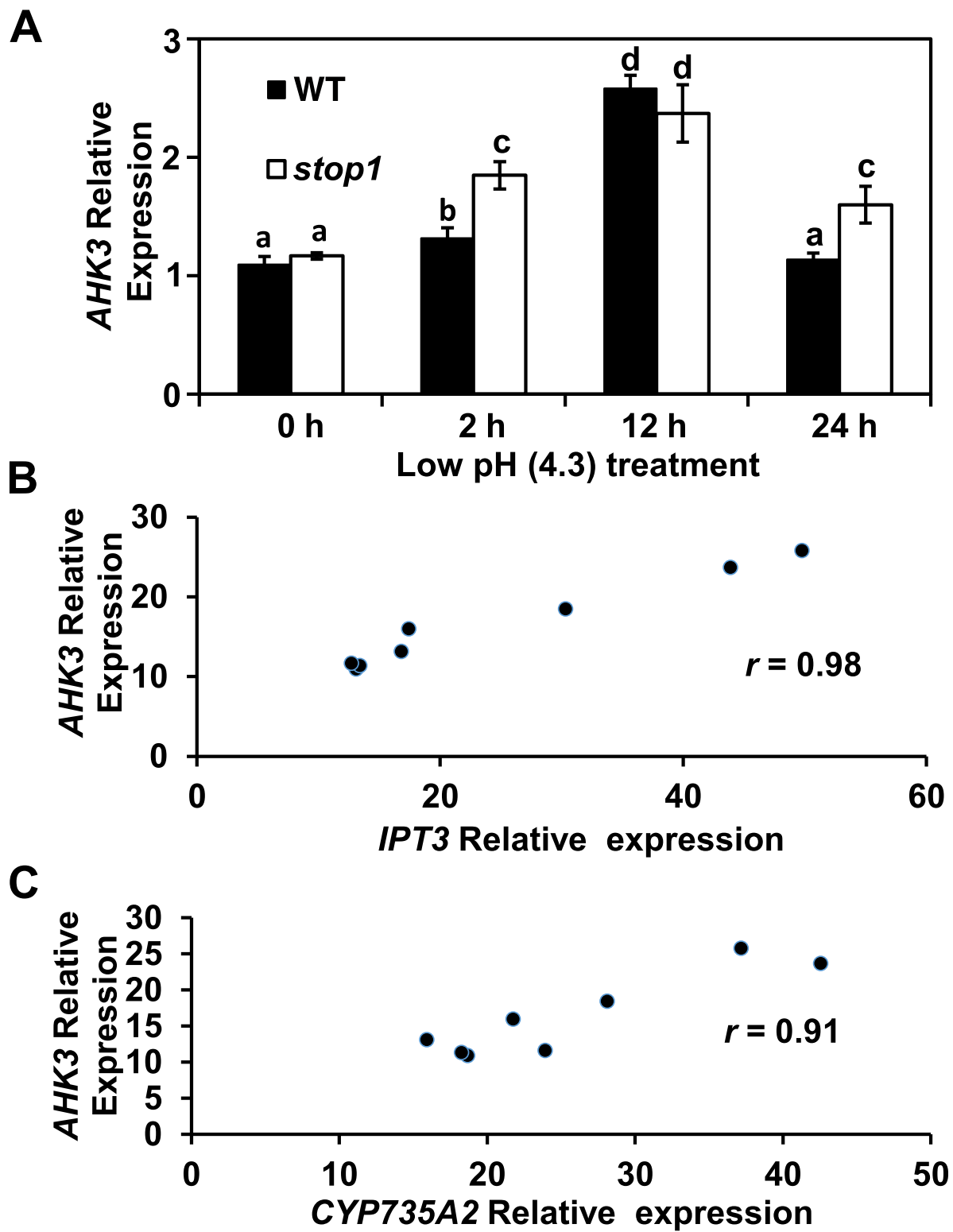

Fig. 5 AHK3 expression is induced by low pH (4.3, A) and is correlated with the expression of IPT3 (B) and CYP735A2 (C). Five-day-old seedlings germinated and grown hydroponically in $1 / 2 \times$ MS solution $(\mathrm{pH}$ 5.8) were transferred to a new low- $\mathrm{pH}$ (4.3) hydroponic solution for indicated durations. Root tissues were collected for RNA extraction and subsequent real-time RT-qPCR analyses. Data are means \pm SD $(n=3)$. ${ }^{*}$, significant differences between the stop 1 mutant and the WT $(p<0.05)$

indicated that under the low-pH (4.3) stresses, the stop1 mutant had stronger and longer-lasting AHK3 expression than the WT. Correlation analysis revealed that the expression levels of $A H K 3$ were highly and positively correlated with those of the other two cytokinin-promoting genes, IPT3 and CYP735A2, with $r=0.98,0.91$, respectively (Fig. 5B, C). In contrast, the expression of the other two cytokinin receptor genes, $A H K 2$ and $A H K 4$, was slightly induced by the low-pH treatment and showed no significant differences in gene expression levels between WT and stop1 (Fig. S3).
To investigate the possible involvement of $A R R \mathrm{~s}$ in cytokinin- and STOP1-mediated low-pH resistance, we examined the expression patterns of seven cytokinininducible type-A $A R R$ s in the root. In general, under the control (pH 5.8, $0 \mathrm{~h}$ ) condition, root expression of ARR4, $A R R 5$, and $A R R 6$ was considerably higher than those of ARR7, ARR8, ARR15, and ARR16 in the WT and the stop1 mutant (Fig. 6).

Furthermore, in the WT, a low-pH (4.3) treatment significantly increased the expression of ARR5 and ARR16, whereas the expression of the rest type-A ARRs either 


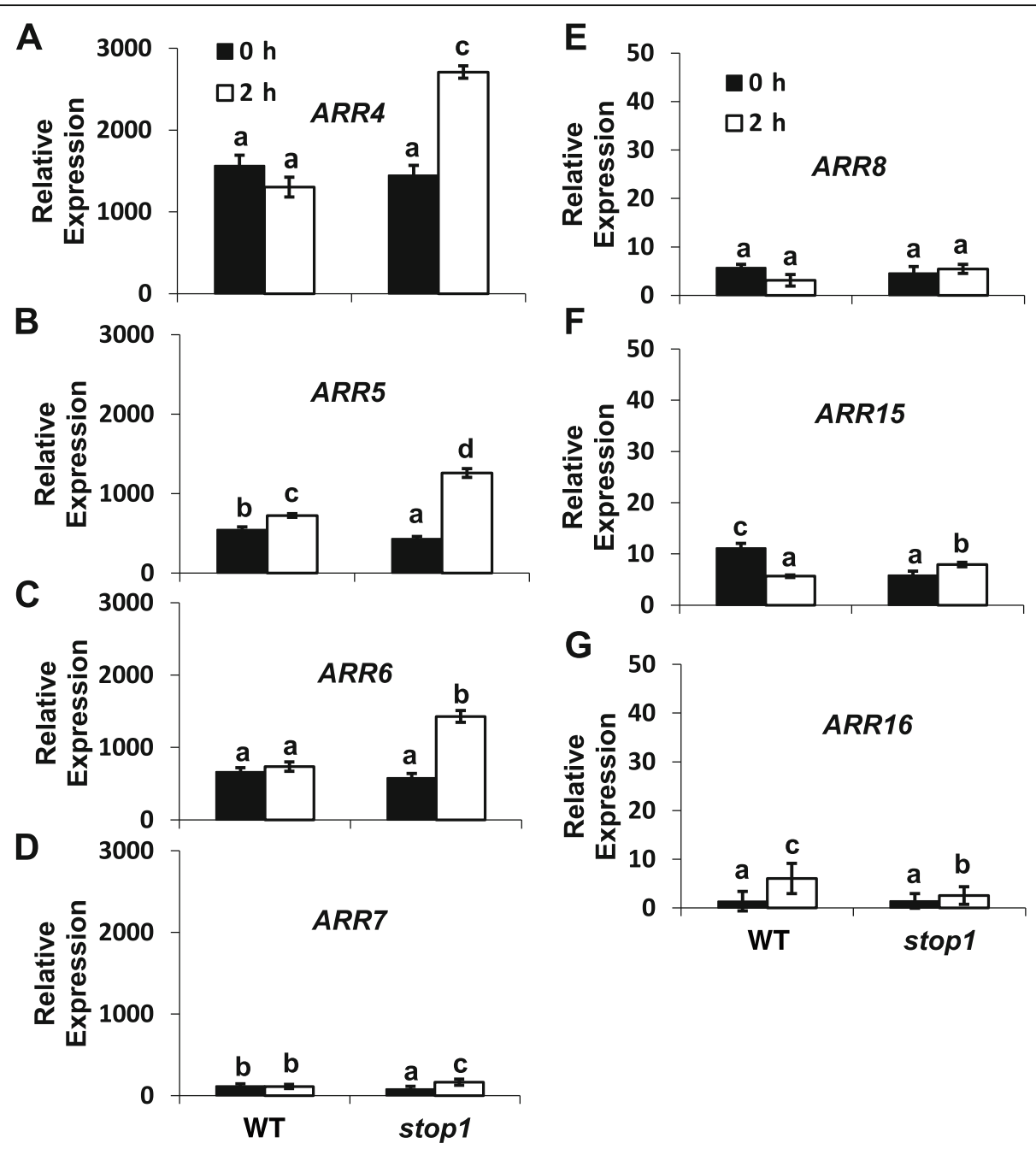

Fig. 6 Relative expression of ARR4 (A), ARR5 (B), ARR6 (C), ARR7 (D), ARR8 (E), ARR15 (F), and ARR16 (G) in WT and stop1 with or without $2 \mathrm{~h}$ low $\mathrm{pH}$ (4.3) treatment. Five-day-old seedlings germinated and grown in $1 / 2 \times \mathrm{MS}$ solution ( $\mathrm{pH}$ 5.8) were treated in a new low-pH solution for $2 \mathrm{~h}$. Root tissues were collected for RNA extraction and subsequent real-time RT-qPCR analyses. Data are means \pm SD $(n=3)$. Different letters indicate significant differences $(p<0.05)$ between different samples

remained unchanged or slightly declined in the case of ARR15 expression (Fig. 6). These results indicate that the low-pH treatment barely impacts the expression of the cytokinin up-regulated type-A ARRs in the WT.

In contrast, the low-pH treatment quickly induced the expression of ARR4, ARR5, and ARR6, the three highly expressed and cytokinin-inducible type-A ARRs, in the root of stop1 (Fig. 6). Therefore, low pH mimics cytokinins in inducing root expression of ARR4, ARR5, and ARR6 in stop1.

\section{Discussion}

STOP1 suppresses the unknown factors that negatively affect root growth and development under low-pH stresses

It has been well documented that STOP1 is regulated at posttranslational but transcriptional levels in Arabidopsis. For instance, STOP1 transcript levels remained unchanged upon low-pH stresses (Iuchi et al., 2007). However, the STOP1 protein level remains low under normal growth conditions ( $\mathrm{pH}$ 5.8) but is significantly enhanced under the low-pH and aluminum stresses via posttranslational regulations (Zhang et al., 2019; Fang et al., 2020). In this report, we demonstrated that STOP1 promoted cytokinin signaling outputs, influencing root meristem size and lateral and adventitious root formation (Figs. 2, 3; Table 1). Therefore, the lower STOP1 level at the normal $\mathrm{pH}$ will presumably contribute less to promoting cytokinin signaling outputs in the root as proposed in a working model for the STOP1-mediated and cytokinin-involved low-pH resistance network in Arabidopsis (Fig. 7A). In the root, cytokinins act antagonistically with auxins to define the boundary of auxin maximum, i.e., the border of the root meristem in the root tip, and delimit the positions of lateral roots along 

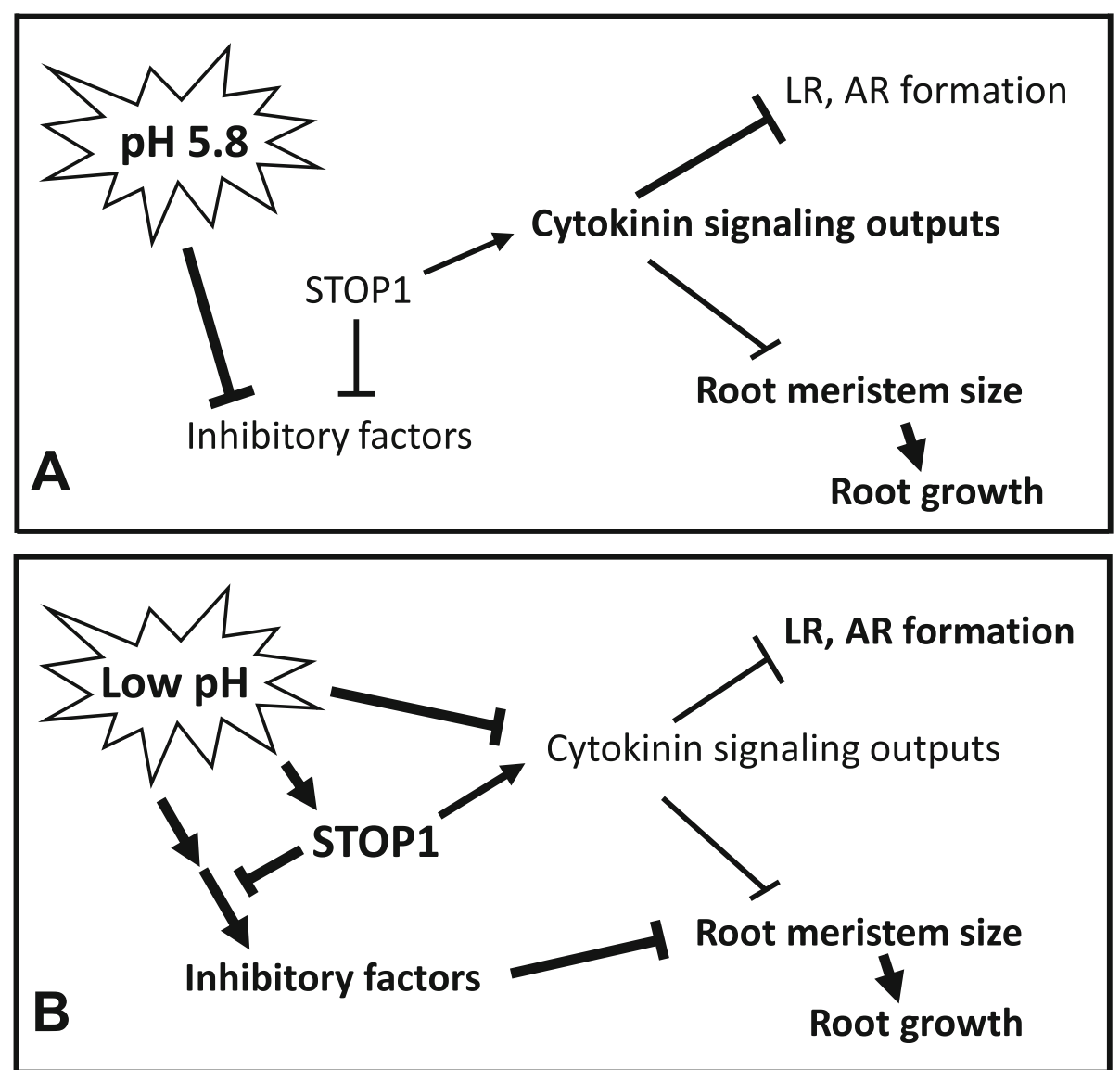

Fig. 7 A working model for the STOP1-mediated and cytokinin-involved resistance to low-pH stresses. A The root-growth inhibitory factors are suppressed by normal pH. The STOP1 protein level remains low. The cytokinin signaling outputs are involved in defining the root meristem boundary, i.e.i.e., the root meristem size, and inhibiting lateral root and adventitious root formation. The root meristem size is a key factor influencing root growth. B The root growth inhibitory factors are activated at low pH, which strongly suppresses root meristem size. STOP1 is activated by proton stresses, presumably at posttranslational levels, which represses the inhibitory factors but promotes the cytokinin signaling outputs. Arrows indicate promotion. T bars indicate inhibition

the primary root and the adventitious roots on the stem (Werner et al., 2003; Ioio et al., 2008; Bielach et al., 2012). Thus, at normal $\mathrm{pH}$, root cell division and differentiation activities are well balanced by the interactions between auxins and cytokinins.

The stop 1 null mutant is highly susceptible to proton stresses (Fig. 1A) (Iuchi et al., 2007; Jiang et al., 2017), indicating that STOP1 is essential for suppressing the root-growth inhibitory factors, which are turned on upon the proton stresses (Fig. 7B). In the meantime, low-pH (4.3) stresses also inhibited root growth of the WT plant (Fig. 1A), indicating that the STOP1-mediated low-pH resistant mechanism could not wholly offset the deleterious effects of the proton stresses (Fig. 7B).

Low-pH stresses inhibit root growth mainly via reducing the root meristem size, i.e., the root meristem activity, in WT and stop1 (Fig. 2). The reduced root meristem size could result from the unknown low-pH inhibitory factors and enhanced cytokinin signaling outputs, both of which are regulated by STOP1 (Figs. 1; 2A, C; 7B). In addition, STOP1 suppresses the unknown inhibitory factors activated by low-pH stresses (Fig. 7B), as indicated by the highly sensitive phenotype of the stop1 mutant to proton stresses (Fig. 1).

The enhanced formation of lateral root and adventitious roots in stop1 (Table 1) also suggests that the cytokinin signaling outputs were lowered in the stop 1 mutant. In other words, the loss of the wild-type function of STOP1 leads to suppressed cytokinin signaling outputs in the root (Fig. 7). Thus, the cytokinin signaling outputs were suppressed by low-pH stresses but promoted by STOP1 (Figs. 2, 3, 7B).

The overall cytokinin signaling outputs, reflected by the TCSn:GFP intensity, were lower at low $\mathrm{pH}$ than at normal $\mathrm{pH}$ (Figs. 2, 3, 7). These results indicated that, at low $\mathrm{pH}$, the effect of low-pH stresses on suppressing cytokinin signaling outputs outperformed that of the STOP1 on promoting the cytokinin signaling outputs 
(Figs. 3A, E, I, M; 7B). As a result, the overall cytokinin signaling outputs in the roots were decreased at low $\mathrm{pH}$. Furthermore, under the low-pH stresses, the root meristem activities could be promoted by the reduced overall cytokinin signaling outputs but suppressed by the unknown low-pH inhibitory factors (Figs. 1, 2, 7B). The potent root-growth-inhibition phenotypes of the WT and stop1 plants under low-pH stresses indicate that the effects of the inhibitory factors exceed those of the reduced cytokinin signaling outputs in determining root meristem activities (Fig. 7B).

Furthermore, at low $\mathrm{pH}(\mathrm{pH} 4.3)$, the stop 1 root lost its quiescent center (QC) identity (Figs. 2A, S2B). These results indicate that STOP1 is not only required for maintaining root meristem activities, but it also is essential for preserving the root stem-cell niche under proton stresses. However, further studies are necessary to elucidate the molecular bases underlying STOP1's functions in these processes.

\section{Cytokinins are involved in STOP1-mediated resistance to proton stresses}

Cytokinin oxidase/dehydrogenase 1 (CKX1) facilitates the breakdown of cytokinins in the plant cell (Werner et al., 2003). CKX1 overexpression in Arabidopsis lowered endogenous cytokinin concentrations of the transgenic plants to $30-45 \%$ of the wild-type contents, suppressing shoot growth and development but promoting root growth and the formation of lateral and adventitious roots (Werner et al., 2003). These results indicate that cytokinins are positive and negative regulators for the shoot and root development, respectively (Werner et al., 2003; Aloni et al., 2006; Dello Ioio et al., 2007; Chang et al., 2013).

The WT seedlings produced significantly more lateral and adventitious roots at low $\mathrm{pH}$ than at normal $\mathrm{pH}$ (Table 1). Moreover, under the control condition ( $\mathrm{pH}$ 5.8 , -BA), the stop 1 seedlings possessed significantly more lateral and adventitious roots than the WT (Table $1)$. These phenotypes mimic the effect of reduced cytokinin-signaling outputs on promoting lateral and adventitious root formation (Nishimura et al., 2004; Bielach et al., 2012). Significantly, adding $0.5 \mu \mathrm{M}$ BA to the growth media diminished or eliminated the promotional effects of the low-pH stresses and lossof-STOP1 functions on promoting lateral-root and adventitious-root formation (Table 1). Therefore, the enhanced lateral- and adventitious-root formation by low-pH stresses and stop1 could result from reduced cytokinin signaling outputs.

Indeed, our results indicate that the wild-type function of STOP1 boosts cytokinin signaling outputs in the root (Fig. 3), which could lead to suppressed lateral and adventitious root formation (Table 1). In the meantime,
low-pH stresses suppress root cytokinin-signaling outputs (Fig. 3), positively influencing lateral and adventitious root formation (Table 1). Thus, at low pHs, the STOP1 function counteracts the inhibitory effects of low-pH stresses on reducing the cytokinin signaling outputs in the root (Fig. 7), which is essential for preserving root stem-cell identity and root meristem activities upon proton stresses for a reason explained below.

\section{Expression patterns of genes involved in the two- component signaling pathway}

The expression of the cytokinin-promoting genes IPT3, $C Y P 35 A 2$, and $A H K 3$ was induced by low-pH stresses in both the WT and the stop1 mutant (Figs. 4, 5), which could be a compensatory response to the lowered cytokinin-signaling outputs at low pH (Fig. 3). Furthermore, the expression of these cytokinin-promoting genes was induced by the low-pH stresses earlier in stop 1 than in WT (Figs. 4, 5). In contrast, the cytokininbiodegradation gene $C K X 1$ was induced later than the cytokinin-promoting genes, and the steady-state of CKX1 expression lasted longer in stop1 than in WT (Fig. 4). These results are consistent with lower cytokinin signaling outputs in stop1 than in WT under proton stresses (Figs. 2, 3), such that the stop1 mutant requires a higher cytokinin compensation than the WT.

Type-A ARRs are negative regulators of the cytokinin signaling network, responsible for the final realization of the cytokinin signaling (Hwang and Sheen, 2001; Kiba et al., 2003; To et al., 2004; Leibfried et al., 2005). We found that the expression of three cytokinin-inducible type-A ARRs, i.e., ARR4, ARR5, and ARR6, was highly induced by low pH stresses in stop1 but not in WT (Fig. 6), which could contribute to the reduced cytokinin signaling outputs observed in stop1 under proton stresses (Figs. 2, 3).

\section{Basal levels of cytokinins are required for root growth and development}

It has been reported that the cytokinin signaling mutants, ahk3 and arr1, 12, with reduced sensitivity to cytokinins (Dello Ioio et al., 2007) and the cytokinin deficient mutants with $~ 30-45 \%$ of the wild-type levels of cytokinins (Werner et al., 2003) exhibit accelerated root growth and enhanced formation of lateral and adventitious roots. These results indicate that cytokinins are negative regulators for root growth and development. However, higher-order loss-of-function mutants of the genes encoding cytokinin receptors (AHKs) and Arabidopsis His Phosphotransfer Proteins (AHPs) display reduced root meristem activities (Higuchi et al., 2004; Nishimura et al., 2004; Hutchison et al., 2006), indicating that although high cytokinin concentrations inhibit root growth, basal levels of cytokinin signaling 
outputs are essential for root meristem function (To and Kieber, 2008).

As discussed above, low-pH stresses and the loss-ofSTOP1 function additively suppress the cytokinin signaling outputs in stop1 (Fig. 7). As a result, the cytokinin signaling outputs in the stop 1 mutant could be too low to maintain the root stem-cell niche and root meristem activities under low pH stresses (Fig. 2A, B). This hypothesis is supported by the root phenotype of the WT at low $\mathrm{pH}(4.3,-\mathrm{BA})$ and the root phenotype of stop1 at low $\mathrm{pH}$ with $0.5 \mu \mathrm{M}$ BA. Figures $2 \mathrm{~A}$ and $\mathrm{S} 2 \mathrm{~B}$ show that the root stem-cell niche was lost in the stop 1 mutant, whereas the WT maintained its QC and root stem-cell identity under proton stresses (Fig. S2). Furthermore, supplementation of $0.5 \mu \mathrm{M}$ BA could rescue the root stem-cell identity, as evidenced by the restored QC structure in the root tip (Fig. S2C), and promoted root meristem activities of the stop 1 plant at low pH (Fig. 2A, $\mathrm{B})$. These results suggest that the severe defects in root development and growth of the stop 1 plant are partially due to its extremely low cytokinin signaling outputs in the root. Therefore, it is necessary for STOP1 to counteract the effects of low-pH stresses on reducing cytokinin signaling outputs under proton stresses (Fig. 7B).

\section{Materials and methods}

\section{Plant materials and growth conditions}

The wild-type Arabidopsis thaliana (Columbia ecotype, Col-0) and the stop1 T-DNA insertion line, SALK 114180 (stop1) were acquired from the Arabidopsis Biological Resource Center (ABRC). Seeds were surface sterilized with $70 \%(\mathrm{v} / \mathrm{v})$ ethanol for $10 \mathrm{~min}$ and washed 5 times with sterilized water. After $2 \mathrm{~d}$ of cold treatment $\left(4{ }^{\circ} \mathrm{C}\right)$, seeds were sown on half-strength $(1 / 2 \mathrm{x})$ Murashige and Skoog (MS) medium plates (1.2\% agar, 1\% sucrose, $\mathrm{pH} 5.8$ ) and grown in a growth chamber with a $16 \mathrm{~h}: 8 \mathrm{~h}$, light: dark, cycle at $23^{\circ} \mathrm{C}$ (Hoekenga et al., 2006; Jiang et al., 2017; Wang et al., 2017; Wang et al., 2018; Wang et al., 2021). After $4 \mathrm{~d}$, seedlings were transferred to $1 / 2 \times$ MS plates $(0.8 \%$ gellan gum, $1 \%$ sucrose, $\mathrm{pH} 5.8$ or 4.3) with different concentrations of 6benzyladenine (BA) (Sigma-Aldrich) and grown in the same growth chamber. Then, root growth of 5-d-old seedlings was measured. For hydroponic growth, seeds were germinated and growth on 1/2 x MS solutions at pH 5.8 or 4.3 for $5 \mathrm{~d}$.

\section{Measurement of lateral root density and adventitious root density}

Total lateral roots and adventitious roots were counted for individual 6-d-old seedlings germinated and grown on $1 / 2$ x MS medium ( $\mathrm{pH} 5.8$ or 4.3) plates supplemented without or with $0.5 \mu \mathrm{M}$ BA. Lateral root density was defined as the number of lateral roots per $\mathrm{cm}$ of the primary root, and adventitious root density was defined as the number of adventitious roots per plant.

\section{Root length and meristem size analyses}

Seeds were germinated and grown on $1 / 2 \times$ MS agar plates ( $\mathrm{pH} 5.8)$ in a growth chamber with $16 \mathrm{~h}: 8 \mathrm{~h}$, light: dark $\left(23^{\circ} \mathrm{C}\right)$ for $4 \mathrm{~d}$. Then, the 4 -d-old seedlings were transferred to $1 / 2 \times$ MS gellan gum plates $(\mathrm{pH} 5.8$ or 4.3) supplemented without or with $0.5 \mu \mathrm{M}$ BA for a 16-h treatment in a growth chamber with $16 \mathrm{~h}: 8 \mathrm{~h}$, light: dark $\left(23^{\circ} \mathrm{C}\right)$. The treated seedlings were then cleared as described (Malamy and Benfey, 1997). Subsequently, the cleared root samples were observed with a DM5500B Leica Differential Interference Contrast (DIC) microscope under a 20x water immersion objective. Each experiment contained a minimum of 25 plants. Experiments were repeated twice independently. Root meristem size is defined as the number of cortex cells in a file from the quiescent center to the first elongated cortex cell (Perilli and Sabatini, 2010). For measuring root length, plates were photographed. Then, the root images were analyzed by the Image J software (https://imagej.nih.gov/ij/).

\section{Monitoring cytokinin-signaling outputs with the TCSn:GFP reporter}

The Two-Component signaling Sensor new (TCSn):green fluorescent protein (GFP) construct (Zürcher et al., 2013) was stably transformed into the WT (Col- 0$)$ and the stop1 mutant. Transgenic TCSn:GFP lines (T2) exhibited strong GFP expression patterns that match the known cytokinin responses and functions were chosen for further studies. Four-day-old seedlings germinated on vertical half-strength $(1 / 2 \mathrm{x})$ MS agar plates $(\mathrm{pH} 5.8)$ were transferred to $1 / 2 \times$ MS gellan gum plates $(\mathrm{pH} 4.3$ or 5.8), supplemented with or without $0.5 \mu \mathrm{M}$ BA for a $16 \mathrm{~h}$ treatment (Jiang et al., 2017). Subsequently, the seedlings and their GFP fluorescent patterns were observed under a Leica Laser Microdissection 7 (LMD7) microscope.

\section{RNA isolation and real-time quantitative RT-qPCR}

About $10 \mathrm{mg}$ of surface-sterilized seeds were germinated in Magenta boxes containing sterile hydroponic growth solution (1/2 x MS) ( $\mathrm{pH} 5.8)$ in a growth chamber with a $16 \mathrm{~h}$ light: $8 \mathrm{~h}$ dark cycle at $23^{\circ} \mathrm{C}$ (Hoekenga et al., 2006; Wang et al., 2017). After $5 \mathrm{~d}$, seedlings were treated with new low-pH (4.3) hydroponic growth solutions for indicated durations. No differences in responses of the WT and stop 1 plants to low-pH stresses were found when the treatments were conducted on agar plates or in hydroponic solutions (Fig. S1).

Total RNAs were extracted from root tissues with the RNeasy Mini Kit (Qiagen) according to the manufacturer's instructions. First-strand cDNAs were synthesized from $5 \mu \mathrm{g}$ DNase I-digested total RNAs using the 
SuperScriptIII First-Strand Synthesis System (Invitrogen). Real-time RT-qPCR was performed with a 7500 Fast Real-Time PCR System (Applied Biosystems, Inc.) according to manufacturers' protocols. The relative expression levels of the target genes were referred to an endogenous calibrator gene, $18 \mathrm{~S}$ rRNA. The sequences of the RT-qPCR primers are listed in Table S1.

\begin{abstract}
Abbreviations
AHK: Arabidopsis Histidine Kinase; AHP: Arabidopsis His phosphotransfer Protein; ALMT1: Aluminum-activated malate transporter 1; ALS3: Aluminum sensitive 3; ARR: Arabidopsis response regulator; BA: 6-benzyladenine; CKX: Cytokinin oxidase/dehydrogenases; CYP735A2: Cytochrome P450 monooxygenase 735A2; GUS: $\beta$-glucuronidase; IPT3: Isopentenyl transferase 3; MATE: Multidrug and toxic compound extrusion; RR: Response regulators; RRG\%: Relative root growth; STOP1: Sensitive to proton rhizotoxicity1; TCS: Two-component signaling
\end{abstract}

\section{Supplementary Information}

The online version contains supplementary material available at https://doi. org/10.1007/s44154-022-00033-6.

Additional file 1: Fig. S1. The effects of agar and hydroponic media on the root growth of wild type and stop1. Figure S2. The loss of root stem-cell niche in stop 1 under low-pH stresses. Figure S3. The effects of low pH treatment on expression of AHK2 and AHK4. Table S1. Sequences of real-time RT-qPCR primers.

\section{Acknowledgments}

We thank professor Bruno Müller of the University of Zurich for providing the TCSn::GFP construct; Mamta Srivastava of the Plant Cell Imaging Center of the Boyce Thompson Institue image facility and John Ramsey of the Robert W. Holley Center, USDA-ARS for the help of microscopic analyses.

\section{Authors' contributions}

J.L. conceived, projected, guided and performed the experiments, and wrote the article; F.J. designed and performed the experiments, analyzed the data, and wrote the paper; S.M.L. performed the experiments and analyzed the data; T.S., L. L., and T.W. analyzed the data and reviewed and edited the article. All authors have read and approved the final manuscript.

\section{Funding}

This work is supported by funds from the US Department of AgricultureAgricultural Research Service (to $\mathrm{JL}$ ) and the National Key Research and Development Program of China (2016YFD0102000) (to TW).

\section{Availability of data and materials}

The data supporting this study's findings are available from the

corresponding author, $J$, upon reasonable request.

\section{Declarations}

Ethics approval and consent to participate

Not applicable.

\section{Competing interests}

The authors declare that they have no conflict of interest.

\section{Author details}

${ }^{1}$ Robert W. Holley Center for Agriculture and Health, USDA-ARS, Ithaca, NY 14853, USA. ${ }^{2}$ Chengdu Institute of Biology, Chinese Academy of Sciences, Chengdu, Sichuan, China. ${ }^{3}$ Plant Breeding and Genetics Section, School of Integrative Plant Science, Cornell University, Ithaca, NY 14853, USA.
Received: 9 December 2021 Accepted: 10 January 2022

Published online: 08 March 2022

\section{References}

Aloni R, Aloni E, Langhans M, Ullrich C (2006) Role of cytokinin and auxin in shaping root architecture: regulating vascular differentiation, lateral root initiation, root apical dominance and root gravitropism. Ann Bot 97(5):883893. https://doi.org/10.1093/aob/mcl027

Arqueso CT, Ferreira FJ, Kieber JJ (2009) Environmental perception avenues: the interaction of cytokinin and environmental response pathways. Plant Cell Environ 32(9):1147-1160. https://doi.org/10.1111/j.1365-3040.2009.01940.x

Baluška F, Mancuso S, Volkmann D, Barlow PW (2010) Root apex transition zone: a signalling-response nexus in the root. Trends Plant Sci 15(7):402-408. https://doi.org/10.1016/j.tplants.2010.04.007

Bielach A, Podlešáková K, Marhavý P, Duclercq J, Cuesta C, Müller B, Grunewald W, Tarkowski P, Benková E (2012) Spatiotemporal regulation of lateral root organogenesis in Arabidopsis by cytokinin. Plant Cell 24(10):3967-3981. https://doi.org/10.1105/tpc.112.103044

Brenner WG, Ramireddy E, Heyl A, Schmülling T (2012) Gene regulation by cytokinin in Arabidopsis. Front Plant Sci 3:8. https://doi.org/10.3389/fpls.2012. 00008

Casamitjana-Martınez E, Hofhuis HF, Xu J, Liu C-M, Heidstra R, Scheres B (2003) Root-specific CLE19 overexpression and the sol1/2 suppressors implicate a CLV-like pathway in the control of Arabidopsis root meristem maintenance. Curr Biol 13(16):1435-1441. https://doi.org/10.1016/S0960-9822(03)00533-5

Chang L, Ramireddy E, Schmülling T (2013) Lateral root formation and growth of Arabidopsis is redundantly regulated by cytokinin metabolism and signalling genes. J Exp Bot 64(16):5021-5032. https://doi.org/10.1093/jxb/ert291

Cheng C-Y, Kieber JJ (2014) Cytokinin signaling in plants. Molecular Biology. New York: Springer. pp 269-289

Dello loio R, Linhares FS, Scacchi E, Casamitjana-Martinez E, Heidstra R, Costantino P, Sabatini S (2007) Cytokinins determine Arabidopsis rootmeristem size by controlling cell differentiation. Curr Biol 17(8):678-682. https://doi.org/10.1016/j.cub.2007.02.047

Fang Q, Zhang J, Zhang Y, Fan N, van den Burg HA, Huang C-F (2020) Regulation of aluminum resistance in Arabidopsis involves the SUMOylation of the zinc finger transcription factor STOP1. Plant Cell 32(12):3921-3938. https://doi. org/10.1105/tpc.20.00687

Ferreira FJ, Kieber JJ (2005) Cytokinin signaling. Curr Opin Plant Biol 8(5):518-525. https://doi.org/10.1016/j.pbi.2005.07.013

Flores S, Tobin EM (1986) Benzyladenine modulation of the expression of two genes for nuclear-encoded chloroplast proteins in Lemna gibba: apparent post-transcriptional regulation. Planta 168(3):340-349. https://doi.org/10.1007/ BF00392359

Ha S, Vankova R, Yamaguchi-Shinozaki K, Shinozaki K, Tran L-SP (2012) Cytokinins: metabolism and function in plant adaptation to environmental stresses. Trends Plant Sci 17(3):172-179. https://doi.org/10.1016/j.tplants.2011.12.005

Higuchi M, Pischke MS, Mähönen AP, Miyawaki K, Hashimoto Y, Seki M, Kobayashi M, Shinozaki K, Kato T, Tabata S (2004) In planta functions of the Arabidopsis cytokinin receptor family. Proc Natl Acad Sci 101(23):8821-8826. https://doi.org/10.1073/pnas.0402887101

Hirose N, Takei K, Kuroha T, Kamada-Nobusada T, Hayashi H, Sakakibara H (2008) Regulation of cytokinin biosynthesis, compartmentalization and translocation. J Exp Bot 59(1):75-83. https://doi.org/10.1093/jxb/erm157

Hoekenga OA, Maron LG, Piñeros MA, Cançado GM, Shaff J, Kobayashi Y, Ryan PR, Dong B, Delhaize E, Sasaki T (2006) AtALMT1, which encodes a malate transporter, is identified as one of several genes critical for aluminum tolerance in Arabidopsis. Proc Natl Acad Sci 103(25):9738-9743. https://doi. org/10.1073/pnas.0602868103

Hutchison CE, Li J, Argueso C, Gonzalez M, Lee E, Lewis MW, Maxwell BB, Perdue TD, Schaller GE, Alonso JM (2006) The Arabidopsis histidine phosphotransfer proteins are redundant positive regulators of cytokinin signaling. Plant Cell 18(11):3073-3087. https://doi.org/10.1105/tpc.106.045674

Hwang I, Sheen J (2001) Two-component circuitry in Arabidopsis cytokinin signal transduction. Nature 413(6854):383-389. https://doi.org/10.1038/35096500

Ioio RD, Nakamura K, Moubayidin L, Perilli S, Taniguchi M, Morita MT, Aoyama T, Costantino P, Sabatini S (2008) A genetic framework for the control of cell division and differentiation in the root meristem. Science 322(5906):13801384. https://doi.org/10.1126/science.1164147

luchi S, Koyama H, luchi A, Kobayashi Y, Kitabayashi S, Kobayashi Y, Ikka T, Hirayama T, Shinozaki K, Kobayashi M (2007) Zinc finger protein STOP1 is 
critical for proton tolerance in Arabidopsis and coregulates a key gene in aluminum tolerance. Proc Natl Acad Sci 104(23):9900-9905. https://doi.org/ 0.1073/pnas.0700117104

Jiang F, Wang T, Wang Y, Kochian LV, Chen F, Liu J (2017) Identification and characterization of suppressor mutants of stop1. BMC Plant Biol 17(1):128. https://doi.org/10.1186/s12870-017-1079-2

Kiba T, Yamada H, Sato S, Kato T, Tabata S, Yamashino T, Mizuno T (2003) The type-a response regulator, ARR15, acts as a negative regulator in the cytokinin-mediated signal transduction in Arabidopsis thaliana. Plant Cell Physiol 44(8):868-874. https://doi.org/10.1093/pcp/pcg108

Kieber JJ, Schaller GE (2018) Cytokinin signaling in plant development. Development 145:dev149344

Koyama H, Wu L, Agrahari RK, Kobayashi Y (2021) STOP1 regulatory system: centered on multiple stress tolerance and cellular nutrient management. Mol Plant 14(10):1615-1617. https://doi.org/10.1016/j.molp.2021.08.014

Leibfried A, To JP, Busch W, Stehling S, Kehle A, Demar M, Kieber JJ, Lohmann JU (2005) WUSCHEL controls meristem function by direct regulation of cytokinin-inducible response regulators. Nature 438(7071):1172-1175. https:// doi.org/10.1038/nature04270

Liu J, Luo X, Shaff J, Liang C, Jia X, Li Z, Magalhaes J, Kochian LV (2012) A promoter-swap strategy between the AtALMT and AtMATE genes increased Arabidopsis aluminum resistance and improved carbon-use efficiency for aluminum resistance. Plant J 71(2):327-337. https://doi.org/10.1111/j.1365-313 X.2012.04994.X

Liu J, Magalhaes JV, Shaff J, Kochian LV (2009) Aluminum-activated citrate and malate transporters from the MATE and ALMT families function independently to confer Arabidopsis aluminum tolerance. Plant J 57(3):389 399. https://doi.org/10.1111/j.1365-313X.2008.03696.x

Mok DW, Mok MC (2001) Cytokinin metabolism and action. Annu Rev Plant Biol 52(1):89-118. https://doi.org/10.1146/annurev.arplant.52.1.89

Müller B (2011) Generic signal-specific responses: cytokinin and contextdependent cellular responses. J Exp Bot 62(10):3273-3288. https://doi.org/1 $0.1093 / j x b / e r q 420$

Müller B, Sheen J (2008) Cytokinin and auxin interaction in root stem-cell specification during early embryogenesis. Nature 453(7198):1094-1097. https://doi.org/10.1038/nature06943

Nishimura C, Ohashi Y, Sato S, Kato T, Tabata S, Ueguchi C (2004) Histidine kinase homologs that act as cytokinin receptors possess overlapping functions in the regulation of shoot and root growth in Arabidopsis. Plant Cell 16(6): 1365-1377. https://doi.org/10.1105/tpc.021477

O'Brien JA, Benková E (2013) Cytokinin cross-talking during biotic and abiotic stress responses. Front Plant Sci 4:451. https://doi.org/10.3389/fpls.2013.00451

Perilli S, Perez-Perez JM, Di Mambro R, Peris CL, Díaz-Triviño S, Del Bianco M, Pierdonati E, Moubayidin L, Cruz-Ramírez A, Costantino P (2013) RETINOBLASTOMA-RELATED protein stimulates cell differentiation in the Arabidopsis root meristem by interacting with cytokinin signaling. Plant Cell 25(11):4469-4478. https://doi.org/10.1105/tpc.113.116632

Perilli S, Sabatini S (2010) Analysis of root meristem size development. In: Plant Developmental Biology. New York: Springer. pp 177-187

Sadhukhan A, Kobayashi Y, luchi S, Koyama H (2021) Synergistic and antagonistic pleiotropy of STOP1 in stress tolerance. Trends Plant Sci 26(10):1014-1022. https://doi.org/10.1016/j.tplants.2021.06.011

Sawaki Y, luchi S, Kobayashi Y, Kobayashi Y, Ikka T, Sakurai N, Fujita M, Shinozaki K, Shibata D, Kobayashi M (2009) STOP1 regulates multiple genes that protect Arabidopsis from proton and aluminum toxicities. Plant Physiol 150(1):281-294. https://doi.org/10.1104/pp.108.134700

To JP, Haberer G, Ferreira FJ, Deruere J, Mason MG, Schaller GE, Alonso JM, Ecker JR, Kieber JJ (2004) Type-a Arabidopsis response regulators are partially redundant negative regulators of cytokinin signaling. Plant Cell 16(3):658671. https://doi.org/10.1105/tpc.018978

To JP, Kieber JJ (2008) Cytokinin signaling: two-components and more. Trends Plant Sci 13(2):85-92. https://doi.org/10.1016/j.tplants.2007.11.005

Tran L-SP, Urao T, Qin F, Maruyama K, Kakimoto T, Shinozaki K, YamaguchiShinozaki K (2007) Functional analysis of AHK1/ATHK1 and cytokinin receptor histidine kinases in response to abscisic acid, drought, and salt stress in Arabidopsis. Proc Natl Acad Sci 104(51):20623-20628. https://doi.org/10.1073/ pnas.0706547105

Wang Y, Cai Y, Cao Y, Liu J (2018) Aluminum-activated root malate and citrate exudation is independent of NIP1; 2-facilitated root-cell-wall aluminum removal in Arabidopsis. Plant Signal Behav 13(1):e1422469. https://doi.org/1 0.1080/15592324.2017.1422469
Wang Y, Li R, Li D, Jia X, Zhou D, Li J, Lyi SM, Hou S, Huang Y, Kochian L, Liu J (2017) NIP1;2 is a plasma membrane-localized transporter mediating aluminum uptake, translocation and tolerance in Arabidopsis. Proc Natl Acad Sci 114:5047-5052

Wang Y, Xiao E, Wu G, Bai Q, Xu F, Ji X, Li C, Li L, Liu J (2021) The roles of selectivity filters in determining aluminum transport by AtNIP1; 2. Plant Signal Behav 1991686. https://doi.org/10.1080/15592324.2021.1991686

Werner T, Motyka V, Laucou V, Smets R, Van Onckelen H, Schmülling T (2003) Cytokinin-deficient transgenic Arabidopsis plants show multiple developmental alterations indicating opposite functions of cytokinins in the regulation of shoot and root meristem activity. Plant Cell 15(11):2532-2550. https://doi.org/10.1105/tpc.014928

Werner T, Motyka V, Strnad M, Schmülling T (2001) Regulation of plant growth by cytokinin. Proc Natl Acad Sci 98(18):10487-10492. https://doi.org/10.1073/ pnas. 171304098

Zhang Y, Zhang J, Guo J, Zhou F, Singh S, Xu X, Xie Q, Yang Z, Huang C-F (2019) F-box protein RAE1 regulates the stability of the aluminum-resistance transcription factor STOP1 in Arabidopsis. Proc Natl Acad Sci 116(1):319-327. https://doi.org/10.1073/pnas.1814426116

Zürcher E, Tavor-Deslex D, Lituiev D, Enkeli K, Tarr PT, Müller B (2013) A robust and sensitive synthetic sensor to monitor the transcriptional output of the cytokinin signaling network in planta. Plant Physiol 161(3):1066-1075

Zwack PJ, Rashotte AM (2015) Interactions between cytokinin signalling and abiotic stress responses. J Exp Bot 66(16):4863-4871. https://doi.org/10.1093/ jxb/erv172

\section{Publisher's Note}

Springer Nature remains neutral with regard to jurisdictional claims in published maps and institutional affiliations. 